\title{
Effect of Helicobacter pylori infection on the first-line treatment outcomes in patients with immune thrombocytopenic purpura
}

\author{
Ali Dogan ${ }^{1}$, Omer Ekinci ${ }^{2}$, and Senar Ebinc ${ }^{3}$ \\ ${ }^{1}$ Department of Hematology, Faculty of Medicine, Yuzuncu Yil University, \\ ${ }^{2}$ University of Health Sciences, Gazi Yasargil Training and Research Hospital, \\ ${ }^{3}$ Department of Medical Oncology, Faculty of Medicine, Dicle University,
}

May 20, 2021

\begin{abstract}
Background: Helicobacter pylori (H. pylori) eradication therapy is known to increase the platelet count, but in immune thrombocytopenic purpura (ITP), the effect of $\mathrm{H}$. pylori infection on the response to treatment is not clear. This study aims to determine whether the response to the first-line treatment is affected by the states of H. pylori-positivity and -negativity in ITP patients. Methods: Adult newly diagnosed or chronic ITP patients who had not received eradication therapy for H. pylori infection were included. Characteristics of the patients, presence and severity of bleeding, initial platelet count, administered treatments, and treatment response rates were inspected. Results: Of 119 total patients, 32 (26.9\%) were H. pylori-positive, $87(73.1 \%)$ were H. pylori-negative. The most common treatment was standard-dose steroid in both groups $(62.5 \%$ vs $68.9 \%$, $\mathrm{p}=0.524$ ). Rates of complete response, partial response, no response were comparable for the two groups (respectively, $75 \%$ vs $73.6 \%$, and $18.8 \%$ vs $19.5 \%$, and $6.2 \%$ vs $6.9 \%$ ), and there was no significant difference between the groups ( $\mathrm{p}=0.283$ ). Conclusion: It can be stated according to the present study that; in ITP patients in whom treatment is indicated, the response to the first-line treatment without the administration of $\mathrm{H}$. pylori eradication therapy is comparable between $\mathrm{H}$. pylori-positive and H. pylori-negative patients. Keywords: Helicobacter pylori, immune thrombocytopenic purpura, first-line treatment
\end{abstract}

Effect of Helicobacter pylori infection on the first-line treatment outcomes in patients with immune thrombocytopenic purpura

\section{ABSTRACT}

Background: Helicobacter pylori (H. pylori ) eradication therapy is known to increase the platelet count, but in immune thrombocytopenic purpura (ITP), the effect of $H$. pyloriinfection on the response to treatment is not clear. This study aims to determine whether the response to the first-line treatment is affected by the states of $H$. pylori - positivity and -negativity in ITP patients.

Methods: Adult newly diagnosed or chronic ITP patients who had not received eradication therapy for H. pylori infection were included. Characteristics of the patients, presence and severity of bleeding, initial platelet count, administered treatments, and treatment response rates were inspected.

Results: Of 119 total patients, 32 (26.9\%) were H. pylori-positive, 87 (73.1\%) were H. pylori -negative. The most common treatment was standard-dose steroid in both groups $(62.5 \%$ vs $68.9 \%, \mathrm{p}=0.524)$. Rates of complete response, partial response, no response were comparable for the two groups (respectively, $75 \%$ vs $73.6 \%$, and $18.8 \%$ vs $19.5 \%$, and $6.2 \%$ vs $6.9 \%$ ), and there was no significant difference between the groups $(\mathrm{p}=0.283)$.

Conclusion: It can be stated according to the present study that; in ITP patients in whom treatment is indicated, the response to the first-line treatment without the administration of $H$. pylorieradication therapy 
is comparable between $H$. pylori -positive and H. pylori -negative patients.

Keywords: Helicobacter pylori, immune thrombocytopenic purpura, first-line treatment

\section{What's already known about this topic?}

Helicobacter pylori (H. pylori) eradication therapy is known to increase the platelet count, but in immune thrombocytopenic purpura (ITP), the effect of $\mathrm{H}$. pylori infection on the response to treatment is not clear.

\section{What does this article add?}

In this study, presented that ITP patients in whom treatment is indicated, the response to the first-line treatment without the administration of $\mathrm{H}$. pylori eradication therapy is similarly between $\mathrm{H}$. pylori-positive and H. pylori-negative patients.

\section{INTRODUCTION}

Immune (idiopathic) thrombocytopenic purpura (ITP) is an acquired disease characterized by a temporary or permanent decrease in the platelet count that results from the effects of immune-mediated anti-platelet antibodies (1). Its prevalence in adults is approximately $5-10 / 100.000$ and it is more common among females in the adult age group. It is usually associated with a chronic progression and an elevated risk of bleeding due to the severity of thrombocytopenia $(2,3)$.

Thrombocytopenia may be induced by antibodies that are produced in response to pathogen antigens and cross-react with platelets in certain infections, which mainly include viral infections but also consist of bacterial infections. Particularly, lipopolysaccharides in bacteria are reported to bind to the platelet membrane and trigger platelet phagocytosis (4). Similarly, although no mechanism has been proposed to explain how Helicobacter pylori (H. pylori) could be involved in the pathogenesis of immune-mediated platelet destruction, the role of bacterial factors such as the cytotoxin-associated gene A (CagA) protein are currently being investigated (5).

The relationship between H. pylori infection and ITP was first defined in 1998 by Gasbarrini et al. in a study where they reported a high platelet count in 8 of their 11 ITP patients (6). Since then, numerous studies on H. pylori eradication in ITP have been published. However, it is still controversial whether or not $H$. pylori eradication always increases the platelet count in patients diagnosed with ITP. Comprehensive reviews suggest that eradication therapy would need to be considered if a $\mathrm{H}$. pylori infection is detected in patients with typical ITP (7). Studies in the literature have generally focused on the effects of the eradication of $H$. pylori infection on increasing the platelet count in ITP patients and the effects of $H$. pylori positivity on the response to the standard first-line ITP treatment are not clear. The present study aims to determine whether or not the response to the first-line treatment is affected by H. pylori-positive and -negative states in patients diagnosed with ITP.

\section{MATERIALS AND METHODS}

\section{Patients}

A retrospective study in adult patients who were either newly diagnosed with ITP or were under followup for chronic ITP at Yüzüncü Yil University Hospital, Van, Turkey was conducted from January 2010 to March 2019. Follow-up records and data of the patients in the hospital system were retrospectively evaluated. Inclusion criteria included; having a diagnosis of ITP, being older than 18 years, treatment indication and having undergone treatment for ITP, and for those with a H. pylori -positive test result, not having received eradication therapy. Patients who were younger than 18 years, were never treated for ITP, underwent eradication therapy, had missing data were excluded from the study.

The diagnosis of ITP was confirmed according to the Consensus Report on the Investigation and Management of Primary ITP (7). Patients were categorized into two groups: H. pylori -positive and -negative. Fecal antigen testing was used for the diagnosis of $H$. pyloriinfection in all patients. Demographic data of the 
patients at diagnosis, presence and severity of bleeding, initial platelet count, administered treatments, treatment response rates, and post-treatment platelet count were inspected.

The study was approved by the research ethics committee of Yuzuncu Yil University, Faculty of Medicine (date/reference number: 24.01.2018/002). All analyses were performed in accordance with the principles of the Declaration of Helsinki.

\section{Categorization of bleeding symptoms}

The bleeding symptoms were evaluated according to the International Working Group (IWG) bleeding scale. In this scale, bleeding manifestations are grouped into three major domains: skin (S), visible mucosae $(\mathrm{M})$, and organs $(\mathrm{O})$, with a gradation of severity (SMOG). The patients were graded based on physical examination at the time of the visit or on the patient's history and medical reports. The severity of bleeding, as defined in this scale, was graded from 0 to 4 (8).

\section{Evaluation of response after first-line treatment}

Response rates were categorized as complete response, partial response, or no response as follows: a platelet count lower than $30 \times 10^{9} / \mathrm{L}$ and absence of doubling from baseline was considered as no response; a platelet count [?] $30 \times 10^{9} / \mathrm{L}$ but $<100 \times 10^{9} / \mathrm{L}$, and presence of a doubling from baseline and absence of bleeding were considered as a partial response; and a platelet count [?]100x10 $/ \mathrm{L}$ that persisted for 3 months or longer was considered as a complete response (1).

\section{Statistical Analysis}

Statistical analysis of the data was performed using the IBM SPSS 22 (SPSS Inc., Chicago, IL, USA) statistical package program. Descriptive statistics for studied variables (characteristics) were presented as mean, standard deviation, median, minimum and maximum values, and for categorical variables the frequency is expressed as a percentage [n (\%)]. For determining the relationship between groups and categorical variables, the Chi-square test was used. In order to compare H. pylori-positive and H. pylori-negative groups for demographic, clinical and laboratory characteristics, the Man-Whitney U test were performed. The level of significance was set at $p<0.05$.

\section{RESULTS}

\section{Patients' characteristics}

Of the 119 total patients included in the study, 66 (55.5\%) were female. At diagnosis, 32 patients $(26.9 \%)$ were $H$. pylori -positive and $87(73.1 \%)$ were $H$. pylori -negative. The H. pylori-positive and H. pylori negative groups showed a similar median age (32 vs 34) and gender distribution (F/M: 18/14 vs 48/39), with no statistically significant difference in these regards (respectively, $\mathrm{p}=0.127$ and $\mathrm{p}=0.078$ ). Both groups predominantly consisted of newly diagnosed patients and were not significantly different in this aspect $[28(87.5 \%)$ vs $80(91.9 \%), \mathrm{p}=0.094]$, Demographic, clinical and laboratory characteristics of patients with H. pylori positive and $H$. pylori -negative ITP were shown in Table 1.

\section{Bleeding symptoms and platelet counts}

The mean platelet count was determined as $18.4+-22.3 \times 10^{9} / \mathrm{L}$ for the $H$. pylori -positive patient group and as $19.5+-21.8 \times 10^{9} / \mathrm{L}$ for the $H$. pylori -negative patient group; and these groups were not significantly different in terms of platelet count $(\mathrm{p}=0.354)$. Bleeding categories according to the IGW scale were also not significantly different across these groups, with grade 1 bleeding being the most common grade in both groups (for grade 1 bleeding; $56.3 \%$ vs $54.1 \%$, for all grades $\mathrm{p}=0.712$ ) (Table 1 ).

\section{First-line treatment option and response rates}

First-line treatment options and response rates of patients with $H$. pylori-positive and $H$. pylori-negative ITP were shown in Table 2. First-line treatment options offered to all ITP patients were as follows: standarddose steroid or standard-dose steroid plus IVIG or high-dose steroid or high-dose steroid plus IVIG. The 
most common form of treatment was standard-dose steroid (62.5\% vs 68.9\%) in both groups and the groups were not different with regard to treatment options $(\mathrm{p}=0.524)$. Rates of complete response, partial response, and no response after first-line treatment were comparable for the two groups (respectively, $75 \%$ vs $73.6 \%$, and $18.8 \%$ vs $19.5 \%$, and $6.2 \%$ vs $6.9 \%$ ) and there was no significant difference between the groups ( $\mathrm{p}=0.283)$. Median platelet count after first-line treatment was determined as $214 \times 10^{9} / \mathrm{L}$ for $\mathrm{H}$. pylori -positive patients and as $242 \times 10^{9} / \mathrm{L}$ for $H$. pylori -negative patients, with no statistically significant difference $(\mathrm{p}=0.089)$.

\section{DISCUSSION}

The pathogenesis of ITP is not completely clear, and its exact cause has not yet been elucidated. However, the main underlying mechanisms are considered to be an antibody-induced increase in platelet destruction, shortened platelet lifespan and diminished platelet production (9). Although there is no proven mechanism concerning the pathogenesis, certain theories based on the molecular similarity between platelets and $H$. pylori antigens, platelet aggregation, and down-regulation of the reticuloendothelial system have been propounded in an attempt to explain the role of H. pylori in the development of ITP (10).

The prevalence of $H$. pylori infection varies between $22 \%$ and $85 \%$ in patients with chronic ITP (7). There is not enough evidence to support the routine screening of ITP patients for $H$. pylori or the administration of eradication therapy to patients who are $H$. pylori-negative or patients whose $H$. pylori states are unknown (11). The predominant view is that appropriate patients with typical ITP can be administered eradication therapy, both due to its low cost and toxicity, and because it can achieve an increase in the platelet count in H. pylori -positive patients (7). Prospective controlled studies conducted on this matter by Rostami et al. (12) and Suzuki et al. (13) showed that platelet count increased in response to H. pylori eradication and that this was an appropriate therapeutic option for the patients. Following from this, the present study included patients who had never received eradication therapy in order to eliminate potential influence on the targeted treatment outcomes, and compared treatment outcomes between H. pylori-positive and H. pylori -negative patients by assigning them to two different groups.

The primary objective of ITP treatment is to prevent bleeding by raising the platelet count to a constant level. In the absence of bleeding or predisposing comorbid conditions, treatment is rarely required if a platelet count $>50 \times 10^{9} / \mathrm{L}$ is present; and it is reported that treatment must only be considered in symptomatic patients with a platelet count lower than $30 \times 10^{9} / \mathrm{L}$ (14). All patients in this study had symptoms of active bleeding and/or required treatment according to their platelet count.

The treatment involves medications that increase the platelet count by decreasing the scale of platelet destruction through different mechanisms, and immunosuppressive corticosteroid medications constitute the first-line option among these. In patients who have active bleeding or in whom corticosteroid use is contraindicated, intravenous immunoglobulin or anti-D globulin can be used, either in combination with corticosteroids or as a standalone treatment (15). First-line treatment options for ITP are similar for all patients, regardless of whether or not a $H$. pylori infection are present (14). Accordingly, the treatment options received by the two groups in our study were similar and the great majority of the patients had received corticosteroid as the first-line treatment. Where symptoms of bleeding were present and/or a fast increase in platelet count was required, patients received IVIG in combination with standard or high-dose corticosteroids.

The main mechanism underlying the low platelet count seen in $H$. pylori- positive ITP patients is thought to be the destruction of existing platelets by an immune-mediated mechanism rather than a diminution of platelet production. This mechanism was first introduced to the literature by Byrne et al. (16) and later confirmed by another study conducted by Teawtrakul et al. (17). In our study, the response rates achieved by the first-line use of immunosuppressive or immunomodulatory medications were comparable between H.pylori -positive and H.pylori -negative patients. Accordingly, the results we obtained in this study corroborate the mechanism described above.

A review of the literature reveals that previous clinical and review studies and have mainly focused on the effects of eradication therapy on the platelet count response in H.pylori-positive andH.pylori -negative 
ITP patients $(18,19)$. The methods and the results of the present retrospective investigation are distinct from other studies. To the best of our knowledge, the present study is unique as its main component is the direct evaluation of response rates to the first-line treatment in H.pylori -positive and H.pylori -negative ITP patients who did not undergo eradication therapy; and it is valuable in this aspect.

Although the findings of this study may suggest that it is not necessary to routinely screen ITP patients for H.pylori before initiating the first-line treatment, the difference between the groups with respect to recurrence rates and response rates to post-recurrence treatment during long-term follow-up have not yet been elucidated.

In conclusion, although the literature suggests that the platelet count could be increased by standalone eradication therapy without administering conventional ITP treatment, it can be stated based on the present study that response rates to the first-line treatment without eradication therapy are comparable between H.pylori -positive and H.pylori -negative ITP patients in whom treatment is indicated.

\section{Conflict of interest}

The authors of this paper have no conflicts of interests, including specific financial interests, relationships, and/or affiliations relevant to the subject matter or materials included.

\section{Author contributions}

Dogan A was responsible for the accuracy and integrity of the study. Dogan A, Ekinci O and Ebinc S analyzed and interpreted the data, prepared the manuscript, performed the statistical analyses, and were responsible for the final editing.

\section{REFERENCES}

1. Rodeghiero F, Stasi R, Gernsheimer T, Michel M, Provan D, Arnold DM, et al. Standardization of terminology, definitions and outcome criteria in immune thrombocytopenic purpura of adults and children: report from an international working group. Blood . 2009; 113(11):2386-93.

2. Terrell DR, Beebe LA, Neas BR, Vesely SK, Segal JB, George JN. Prevalence of primary immune thrombocytopenia in Oklahoma. Am J Hematol . 2012 Sep;87(9):848-52. doi: 10.1002/ajh.23262.

3. Frederiksen H, Schmidt K. The incidence of idiopathic thrombocytopenic purpura in adults increases with age. Blood . 1999 Aug 1;94(3):909-13. PubMed PMID: 10419881.

4. Semple JW, Aslam R, Kim M, Speck ER, Freedman J. Platelet-bound lipopolysaccharide enhances Fc receptor-mediated phagocytosis of IgG-opsonized platelets. Blood . 2007 Jun 1;109(11):4803-5.

5. Stasi R, Provan D. Helicobacter pylori and Chronic ITP.Hematology Am Soc Hematol Educ Program . 2008:206-11. doi: 10.1182/asheducation-2008.1.206.

6. Gasbarrini A, Franceschi F, Tartaglione R, Landolfi R, Pola P, Gasbarrini G. Regression of autoimmune thrombocytopenia after eradication of Helicobacter pylori. Lancet . 1998 Sep 12; 352(9131):878.

7. Stasi R, Sarpatwari A, Segal JB, Osborn J, Evangelista ML, Cooper N, Provan D, Newland A, Amadori S, Bussel JB. Effects of eradication of Helicobacter pylori infection in patients with immune thrombocytopenic purpura: a systematic review. Blood . 2009 Feb 5;113(6):1231-40. doi: 10.1182/blood-2008-07-167155.

8. Rodeghiero F, Michel M, Gernsheimer T, Ruggeri M, Blanchette V, Bussel JB, et al. Standardization of bleeding assessment in immune thrombocytopenia: report from the International Working Group. Blood . 2013 Apr 4;121(14):2596-606. doi: 10.1182/blood-2012-07-442392.

9. Toltl LJ, Arnold DM. Pathophysiology and management of chronic immune thrombocytopenia: focusing on what matters. Br J Haematol . 2011 Jan;152(1):52-60. doi: 10.1111/j.1365-2141.2010.08412.x.

10. Kuwana M. Helicobacter pylori-associated immune thrombocytopenia: clinical features and pathogenic mechanisms. World J Gastroenterol . 2014 Jan 21;20(3):714-23. doi: 10.3748/wjg.v20.i3.714. 
11. British Committee for Standards in Haematology General Haematology Task Force. Guidelines for the investigation and management of idiopathic thrombocytopenic purpura in adults, children and in pregnancy. Br J Haematol . 2003 Feb;120(4):574-96.

12. Rostami N, Keshtkar-Jahromi M, Rahnavardi M, Keshtkar-Jahromi M, Esfahani FS. Effect of eradication of Helicobacter pylori on platelet recovery in patients with chronic idiopathic thrombocytopenic purpura: a controlled trial. Am J Hematol . 2008 May;83(5):376-81. doi: 10.1002/ajh.21125.

13. Suzuki T, Matsushima M, Masui A, Watanabe K, Takagi A, Ogawa Y, Shirai T, Mine T. Effect of Helicobacter pylori eradication in patients with chronic idiopathic thrombocytopenic purpura-a randomized controlled trial. Am J Gastroenterol . 2005 Jun;100(6):1265-70.

14. Neunert C, Lim W, Crowther M, Cohen A, Solberg L Jr, Crowther MA; American Society of Hematology. The American Society of Hematology 2011 evidence-based practice guideline for immune thrombocytopenia. Blood 2011;117:4190-4207.

15. Provan D, Stasi R, Newland AC, Blanchette VS, Bolton-Maggs P, Bussel JB, Chong BH, Cines DB, Gernsheimer TB, Godeau B, Grainger J, Greer I, Hunt BJ, Imbach PA, Lyons G, McMillan R, Rodeghiero F, Sanz MA, Tarantino M, Watson S, Young J, Kuter DJ. International consensus report on the investigation and management of primary immune thrombocytopenia.Blood . 2010 Jan 14;115(2):168-86. doi: 10.1182/blood-2009-06-225565.

16. Byrne MF, Kerrigan SW, Corcoran PA, Atherton JC, Murray FE, Fitzgerald DJ, Cox DM. Helicobacter pylori binds von Willebrand factor and interacts with GPIb to induce platelet aggregation. Gastroenterology. 2003 Jun;124(7):1846-54.

17. Teawtrakul N, Sawadpanich K, Sirijerachai C, Chansung K, Wanitpongpun C. Clinical characteristics and treatment outcomes in patients with Helicobacter pylori -positive chronic immune thrombocytopenic purpura. Platelets. 2014;25(7):548-51. doi: 10.3109/09537104.2013.841883.)

18. Arnold DM, Bernotas A, Nazi I, Stasi R, Kuwana M, Liu Y, Kelton JG, Crowther MA. Platelet count response to $H$. pylori treatment in patients with immune thrombocytopenic purpura with and without H. pylori infection: a systematic review. Haematologica . 2009 Jun;94(6):850-6. doi: 10.3324/haematol.2008.005348.

19. Franchini M, Cruciani M, Mengoli C, Pizzolo G, Veneri D. Effect ofHelicobacter pylori eradication on platelet count in idiopathic thrombocytopenic purpura: a systematic review and meta-analysis.J Antimicrob Chemother. 2007 Aug;60(2):237-46.

Table 1: Demographic, clinical and laboratory characteristics of patients with ITP

\begin{tabular}{|c|c|c|c|}
\hline Characteristics & $\begin{array}{l}\text { H.pylori-positive } \\
(\mathbf{N}=\mathbf{3 2})\end{array}$ & $\begin{array}{l}\text { H.pylori-negative } \\
(\mathbf{N}=87)\end{array}$ & $\mathbf{P}$ value \\
\hline Age, year & & & 0.127 \\
\hline Median & 32 & 34 & \\
\hline Range & $18-68$ & $18-63$ & \\
\hline Sex, n (\%) & & & 0.078 \\
\hline Female & $18(56.3)$ & $48(55.2)$ & \\
\hline Male & $14(43.7)$ & $39(44.8)$ & \\
\hline $\begin{array}{l}\text { Diagnosis status, } \mathbf{n} \\
(\%)\end{array}$ & & & 0.094 \\
\hline Newly diagnosis & $28(87.5)$ & $80(91.9)$ & \\
\hline Chronic ITP & $4(12.5)$ & $7(8.1)$ & \\
\hline $\begin{array}{l}\text { Baseline platelet } \\
\text { count, } \times 10^{9} / \mathrm{L}\end{array}$ & & & 0.354 \\
\hline Mean \pm SD & $18.4 \pm 22.3$ & $19.5 \pm 21.8$ & \\
\hline
\end{tabular}




\begin{tabular}{llll}
\hline Characteristics & $\begin{array}{l}\text { H.pylori-positive } \\
\mathbf{( N = 3 2 )}\end{array}$ & $\begin{array}{l}\text { H.pylori-negative } \\
\mathbf{( N = 8 7 )}\end{array}$ & P value \\
\hline $\begin{array}{l}\text { Median (range) } \\
\text { Bleeding symptoms, }\end{array}$ & $12(3-28)$ & $14(2-30)$ & 0.712 \\
n (\%) & & & \\
Grade 0 & $7(21.8)$ & $18(20.7)$ & \\
Grade 1 & $18(56.3)$ & $47(54.1)$ & \\
Grade 2 & $5(15.6)$ & $16(18.3)$ & \\
Grade 3 & $2(6.3)$ & $5(5.8)$ & \\
Grade 4 & 0 & $1(1.1)$ & \\
\hline
\end{tabular}

Table 2: First-line treatment options and response rates of patients with ITP

\begin{tabular}{|c|c|c|c|}
\hline Parameters & $\begin{array}{l}\text { H.pylori-positive } \\
(\mathbf{N}=32)\end{array}$ & $\begin{array}{l}\text { H.pylori-negative } \\
(\mathbf{N}=87)\end{array}$ & $\mathbf{P}$ value \\
\hline $\begin{array}{l}\text { First-line treatment } \\
\text { option, n (\%) }\end{array}$ & & & 0.524 \\
\hline Standard-dose steroid & $20(62.5)$ & $60(68.9)$ & \\
\hline $\begin{array}{l}\text { Standard-dose steroid } \\
+ \text { IVIG }\end{array}$ & $6(18.8)$ & $16(18.4)$ & \\
\hline High-dose steroid & $4(12.5)$ & $7(8.1)$ & \\
\hline $\begin{array}{l}\text { High-dose steroid + } \\
\text { IVIG }\end{array}$ & $2(6.2)$ & $4(4.6)$ & \\
\hline $\begin{array}{l}\text { Response rates, } \\
\mathbf{n}(\%)\end{array}$ & & & 0.283 \\
\hline Complete response & $24(75)$ & $64(73.6)$ & \\
\hline Partial response & $6(18.8)$ & $17(19.5)$ & \\
\hline No response & $2(6.2)$ & $6(6.9)$ & \\
\hline $\begin{array}{l}\text { Final platelet count, } \\
\times 10^{9} / \mathrm{L}\end{array}$ & & & 0.089 \\
\hline Median & 214 & 242 & \\
\hline Range & $12-413$ & $7-396$ & \\
\hline
\end{tabular}

